\title{
BERUFLICHE BERATUNG FÜR MENSCHEN 50+ - UNTER BESONDERER BERÜCKSICHTIGUNG DES AGE-MANAGEMENTS IM HUMAN RESOURCE (HR)
}

\section{EINLEITUNG}

Bis heute verbindet man in manchen Ländern mit der Dienstleistung „Berufsberatung" vor allem die Unterstützung junger Menschen bei der Berufswahl, den Übergängen vom Bildungssystem in das Beschäftigungssystem sowie bei der Gestaltung der frühen Berufslaufbahn.

Ein weiteres Merkmal sind nicht immer konstruktive Parallelstrukturen von Bildungsberatung und Berufsberatung sowohl hinsichtlich der theoretischen Grundlagen als auch der Organisation und Qualifizierung der Berater.

Doch in den 1990-er Jahren vollzog sich besonders auf europäischer Ebene ein Wandel hin zu einer lebensbegleitenden beruflichen Beratung, weil man ihre Bedeutung für die Erhaltung der Kompetenz der Arbeitskräfte und damit die wirtschaftliche Stärke eines Landes erkannt hatte.

Einen wesentlichen Anstoß für eine lebensbegleitende Berufsberatung auf europäischer Ebene gab das von OECD und EU-Kommission herausgegebene „Career Guidance: A Handbook for Policy Makers“ (2004).

Dieses Handbuch definiert die in Studien von OECD, EU-Kommission und Weltbank angewandte Definition von Berufsberatung wie folgt (S. 10):

Career guidance refers to services and activities intended to assist individuals, of any Age and at any point throughout their lives, to make

Prof. Dr. BERnD-JOACHIM ERTELT - Institut für Pädagogik, Fakultät für Sozialwissenschaften, Johannes Paul II. Katholische Universität Lublin; ORCID: 0000-0002-4332-1483; Korrespondenzadresse: e-mail: Bernd-Joachim.Ertelt@arbeitsagentur.de 
educational, training and occupational choices and to manage their careers. Such services may be found in schools, universities and colleges, in training institutions, in public employment services, in the workplace, in the voluntary or community sector and in the private sector.

Diese Auffassung ist aus unserer Sicht allerdings zu einseitig auf die individuellen Aspekte der Berufswahl und Laufbahngestaltung ausgerichtet. Daher sollte die Definition ergänzt werden durch das Aufgabenfeld „Beratung im Human Resource Management (HRM) besonders von kleinen und mittleren Betrieben und Organisationen in den Bereichen Personalrekrutierung, Onboarding, Personalentwicklung vor allem durch Qualifizierungsberatung, RetentionsManagement, Outplacement-Beratung und Demografieberatung".

Bemerkenswert ist die eindeutige Aufforderung an die Länder, eine spezifisch auf die Zielgruppe der Älteren ausgerichteten Berufsberatung zu etablieren (OECD / EU-Kommission 2004, S. 35 f.):

- Berufsberatung soll integraler Bestandteil von Angeboten der Erwachsenenbildung in öffentlich finanzierten Einrichtungen für Bildung und Berufsausbildung sein.

- Nutzung wissenschaftlicher Erkenntnisse über den Zusammenhang zwischen aktivem Altern und Gesundheit.

- Etablierung regelmäßiger Kontakte zur Renten- und Sozialversicherung sowie Investmentfonds, um die Verbindung zwischen Finanzplanung, Laufbahnplanung und dem Übergang in den Ruhestand zu analysieren.

- Entwicklung spezieller Berufsberatungsangebote für ältere Menschen durch Outsourcing an Vereinigungen / Organisationen, die bisher schon intensiv mit dieser Zielgruppe arbeiten.

- Nutzung innovativer Techniken zur Information, um möglichst viele Menschen mit ihren individuellen Beratungsbedürfnissen zu erreichen.

- Maßnahmen zur Ausweitung der Dienste der Public Employment Services (PES), um den Beratungsbedürfnissen älterer Menschen gerecht zu werden.

- Förderung von Initiativen von Betrieben und öffentlichen Organisationen, um dem Trend zum vorzeitigen Retirement und zur Langzeitarbeitslosigkeit bei älteren Arbeitnehmern entgegen zu wirken.

- Unterstützung der Betriebe bei der Vorbereitung auf den aktiven Ruhestand. 
- Förderung von Strategien zur Kompetenzentwicklung älterer Mitarbeiter und von Maßnahmen zur Umgestaltung von Arbeitsplätzen in Betrieben unter Einbeziehung der Berufsberatung.

- Qualifizierung von Berufsberatern im Hinblick auf die speziellen Beratungsbedürfnisse Älterer.

Die vom European Lifelong Policy Network (ELGPN) erarbeiteten Leitlinien für lebensbegleitende Beratung (2015) bezieht eine Leitlinie (Nr. 16) auf die „lebensbegleitende Beratung für ältere Menschen“.

Dabei geht es um

- die Entwicklung von Career Management Skills (CMS)

- die Validierung ihrer übertragbaren Kompetenzen und solcher Kompetenzen, die durch non-formale und informelle Bildung erworben wurden

- den Zugang zur Information über Weiterbildungsmöglichkeiten und zu Lernmöglichkeiten

- die individuelle Beratung zu Fragen der Lernmotivation oder zu Möglichkeiten der beruflichen Mobilität.

Dazu werden Merkmale guter Praxis genannt:

- Ältere Erwerbstätige sind eigenständige Zielgruppe im Rahmen der Diversitäts-Politik und bei innovativen Angeboten zum Beispiel in Form von Online-Beratung.

- Maßnahmen zum intergenerativen Wissenstransfer durch systematische Nachfolgeplanung, Einbeziehung älterer Mitarbeiter als Mentoren und Ausbilder.

- Sicherung des Erfahrungswissens Älterer im Rahmen des Wissensmanagements.

- Angebote der öffentlichen Arbeitsverwaltungen (PES) speziell in Berufsberatung und Vermittlung für Ältere.

- Lebensbegleitende Beratung als integraler Bestanteil der Erwachsenenbildung in öffentlich finanzierten Bildungseinrichtungen.

- Berücksichtigung von Anliegen älterer Arbeitnehmer in der Qualifizierung von Beratern und Lehrkräften in der Weiterbildung.

- Anreize für Arbeitgeber, verstärkte Maßnahmen zur Kompetenzentwicklung und zur Erhaltung der Employability von Älteren in ihrem Betrieb anzubieten.

- Weiterentwicklung von Career Management Skills auch bei Älteren, etwa mit Blick auf die Bewältigung von Arbeitslosigkeit und unterwertiger Beschäftigung sowie zur Verbesserung der berufsbezogenen Mobilität. 
- Förderung von Gesundheitsprogrammen für ältere Arbeitnehmer.

- Förderung der Forschung zu lebensbegleitender Beratung für das aktive Altern.

Dazu muss betont werden, dass die Beschäftigungsquote älterer Arbeitnehmer (55-64) in den letzten Jahren stark angestiegen ist, wie die folgende Abbildung 1 zeigt (vgl. Eurofound 2018, S. 6):

Abb. 1: Activity rates of older workers (55-64) by selected countries, EU28, 2017 (in \%)

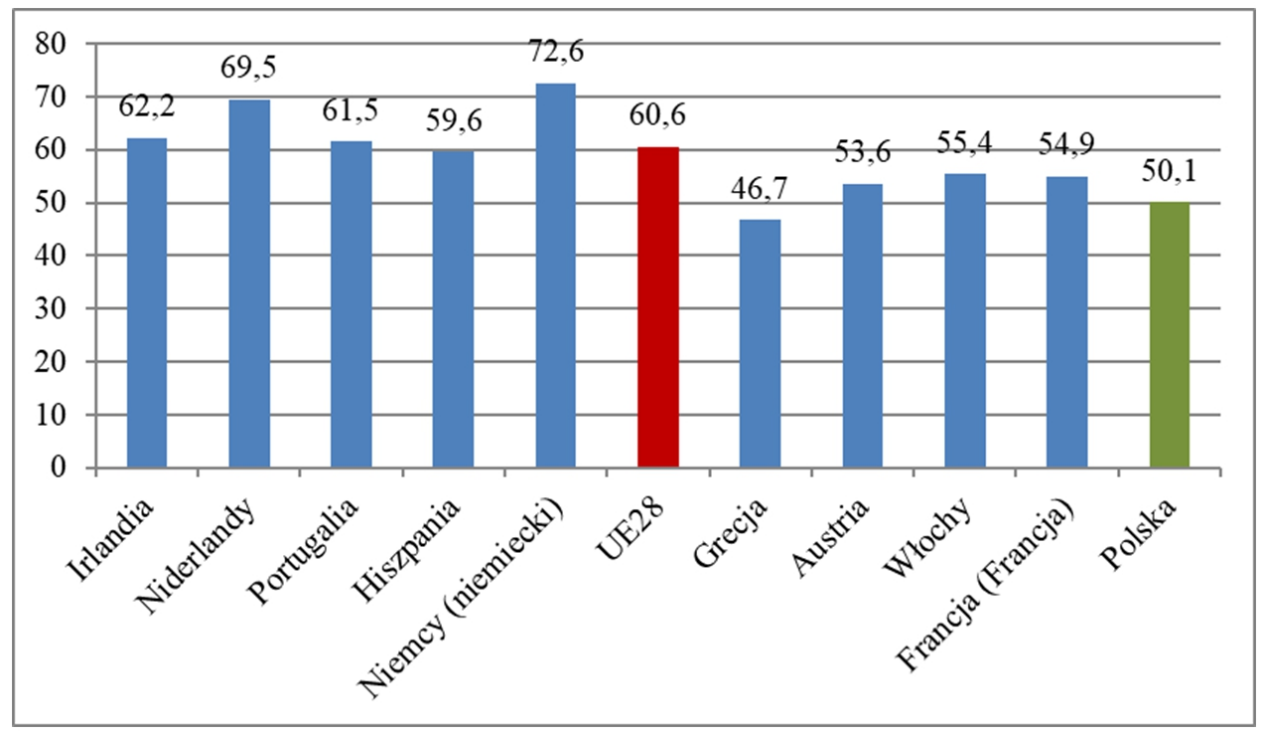

\section{BESTIMMUNG DER ZIELGRUPPEN ÄLTERER}

\subsection{LEBENSPHASENORIENTIERTE MODELLE}

Für die Bestimmung der Zielgruppe Älterer gibt es keine einheitlichen Kriterien. Gesellschaftliche Altersbilder beziehen sich auf gesellschaftliche Deutungsmu-ster und schlagen sich in Altersstereotypen nieder. Diese Altersbilder haben sich im Laufe der Zeit verändert wie folgende Abbildung zeigt. 
Abb. 2: Lebenspannen und Lebensphasen im historischen Vergleich nach Arbinger (1999)
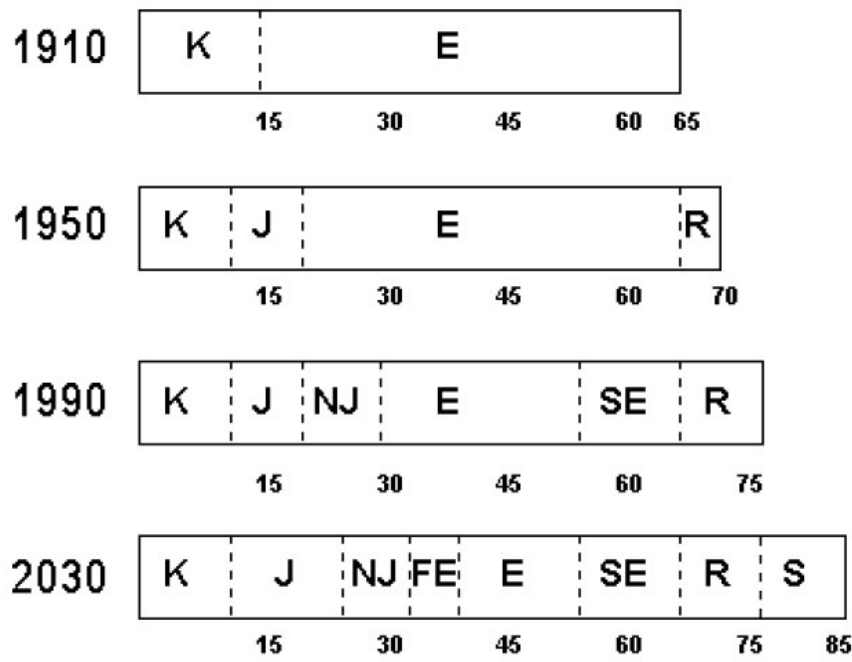

Danach unterschied man um 1910 nur in Kindheit (K) und Erwachsene (E), um 1950 kamen Jugend (J) und Rentner (R) hinzu und um 1990 erweiterte sich die Phaseneinteilung nochmals um Nachjugend (NJ) und Seniorerwachsene (SE).

Die Prognose bis 2030 sieht folgende Differenzierung in acht Lebensphasen voraus:

1. Kindheit (0-13 Jahre)

2. Jugend (14- bis unter 25 Jahre)

3. Nachjugend (15-32 Jahre)

4. Früherwachsene (32-40 Jahre)

5. Erwachsene (40- bis unter 55 Jahre)

6. Seniorerwachsene (55-65 Jahre)

7. Rentner (66-75 Jahre)

8. Senior (76-85 Jahre)

Die Abfolge der Lebensphasen verlaufen nicht immer harmonisch, sondern sind oftmals durch kritische Übergangsereignisse voneinander getrennt. Im Anschluss an Graf (2002; Frey, Ertelt, Ruppert 2017) lassen sich für die Berufsberatung zwei wichtige biografische Ablaufkonzepte unterscheiden: 
a) Das lebenszyklusorientierte Konzept setzt sich zusammen aus dem biosozialen Lebenszyklus, dem familiären Lebenszyklus und dem beruflichen, betrieblichen und stellenbezogenen Lebenszyklus. Die folgende Abbildung 3 zeigt die Entwicklungsschwerpunkte des beruflichen Lebenszyklus (Frey, Ertelt, Ruppert 2017, S. 43 und die hierzu angegebenen Quellen).

Abb. 3: Vier Phasen des beruflichen Lebenszyklus nach Graf 2002

(Lambeck 2008, S. 9)

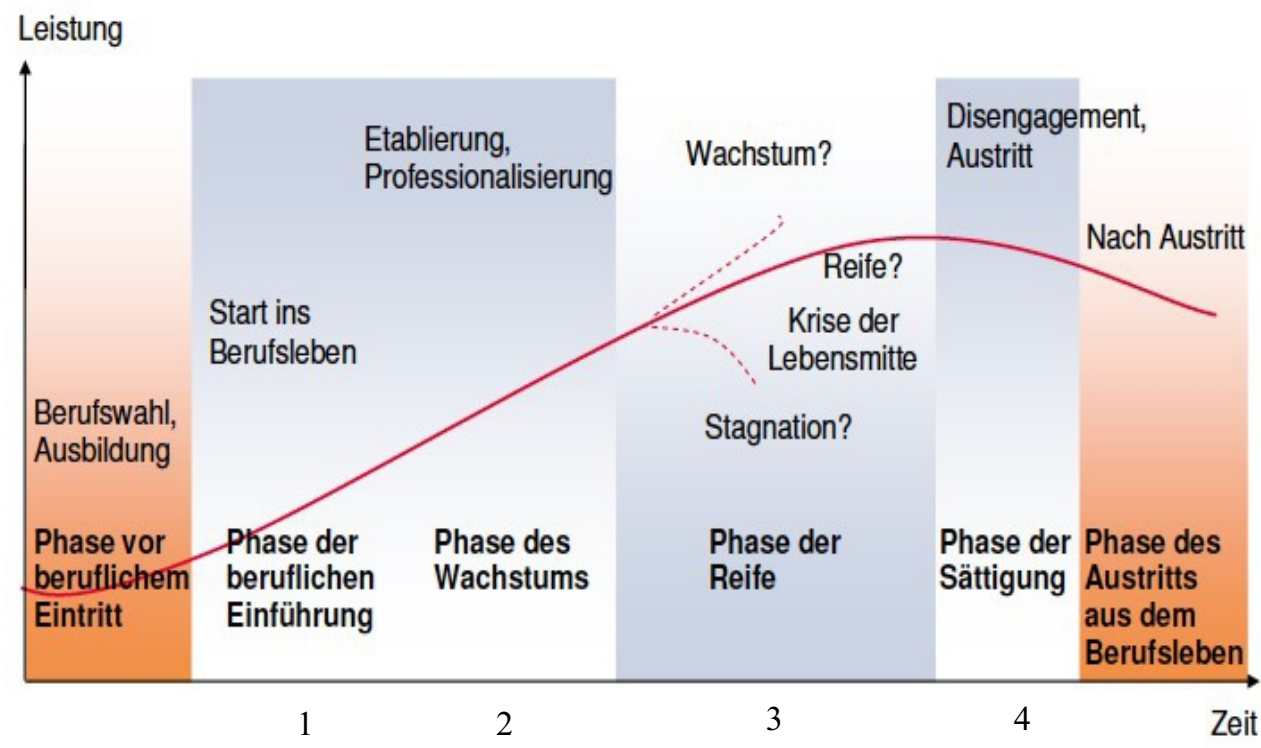

Diese Lebensphasen oder Lebenszyklen haben bestimmte wirtschaftliche und soziale Folgen, wie folgende Tabelle 1 exemplarisch zeigt:

Tab. 1: Entwicklungen im Zusammenhang mit den Lebenszyklen des Menschen (Graf 2001)

\begin{tabular}{|l|l|}
\hline Biosozialer Lebenszyklus & $\begin{array}{l}\text {-Steigende Lebenserwartung } \\
- \text { Entwicklungen im Bereich der Gesundheit }\end{array}$ \\
\hline Familiärer Lebenszyklus & $\begin{array}{l}\text { Entwicklung der Heirats- und Scheidungsrate } \\
- \text { Rückgang der Geburtsrate } \\
- \text { Veränderung der familialen Lebensformen }\end{array}$ \\
\hline Beruflicher Lebenszyklus & - Veränderung der Bedeutung der Arbeit \\
& - Verkürzung des beruflichen Lebenszyklus infolge \\
\hline
\end{tabular}




\begin{tabular}{|l|l|}
\hline & $\begin{array}{l}\text { Verlängerung der Ausbildungszeit und Senkung des } \\
\text { durchschnittlichen Rentenalters } \\
\text { - Veränderungen im Verlauf des beruflichen Lebens- } \\
\text { zyklus (abnehmende Bedeutung der Erstausbildung, } \\
\text { häufigere Arbeitsplatzwechsel, Auftreten von Brü- } \\
\text { chen, Veränderung der bestehenden Arbeitsformen } \\
\text { usw.) }\end{array}$ \\
\hline Betrieblicher Lebenszyklus & $\begin{array}{l}\text { Aberflachung des betrieblichen Lebenszyklus infolge } \\
\text { - Veränderung der Karriereorientierung infolge des } \\
\text { Wertewandels } \\
- \text { Trend zu mehr Selbstverantwortung } \\
- \text { Zunahme der Bedeutung älterer Arbeitnehmer }\end{array}$ \\
\hline Stellenbezogener Lebenszyklus & $\begin{array}{l}\text { - Veränderung der Arbeitsbedingungen } \\
\text { - Veränderung der Arbeitsanforderungen und der } \\
\text { benötigten Qualifikationen }\end{array}$ \\
\hline
\end{tabular}

b) Die lebenslauforientierte Betrachtung findet seit Anfang der 1990er Jahre bei der Analyse der demografischen Entwicklung und der Analyse von Arbeitsmarktprozessen Anwendung, besonders mit Blick auf die Gestaltung lebenslangen Lernens.

Dieses Konzept stellt den biologischen Lebenslauf, Partnerschaft und familiären Lebenslauf, den beruflichen Lebenslauf und betrieblichen Lebenslauf in den Mittelpunkt.

Beide Konzepte, das lebenszyklusorientierte und das lebenslauforientierte, hängen eng miteinander zusammen. Deshalb müsse sie in einem holistischen Beratungsansatz stets gemeinsam betrachtet werden.

Das deutsche Bundesministerium für Familie, Senioren, Frauen und Jugend (2011) konstatiert, dass die „... zunehmende Thematisierung des Alterungsprozesses und des demografischen Wandels ..., zu einer stärkeren Differenzierung gesellschaftlicher Altersbilder geführt [hat]“.

Folgende drei Altersphasen haben sich dabei herauskristallisiert:

1. Junge Alte (50- bis 64-Jährige)

2. Ältere (65- bis 74-Jährige)

3. Hochbetagte (75 Jahre und älter)

$\mathrm{Zu} \mathrm{1:}$ In diese Phase fällt auch der Übergang von dem Erwerbsleben in den Ruhestand. Damit setzt in dieser Zeit eine Umorientierung auf den nachberuflichen Lebensabschnitt ein.

$\mathrm{Zu}$ 2: Die Angehörigen dieser Gruppe investieren einen hohen Anteil ihrer durch den Austritt gewonnenen Zeit in Engagement ihrer Hobby oder eh- 
renamtlichen Aktivitäten. Die „Engagementquote“ stieg in Deutschland in den letzen 20 Jahren deutlich an.

$\mathrm{Zu}$ 3: Diese Gruppe ist durch zunehmende gesundheitliche Probleme in ihren Aktivitäten und ihrem Engagement eingeschränkt. In dieser Altersphase steigt auch der Bedarf an Unterstützung und Pflege.

Doch diese Charakterisierungen sind sehr pauschal, denn es bestehen deutliche Unterschiede hinsichtlich der Aktivitäten in Ehrenämtern oder sozialen Netzwerken, wie folgende Abbildung 4 ausgewählter europäischer Länder zeigt (Bundesministerium für Familie, Senioren, Frauen und Jugend 3/2011, S. 18):

Abb. 4: Ehrenamtliche und im Bereich Netzwerkhilfe informell Engagierte im Ländervergleich (2006)

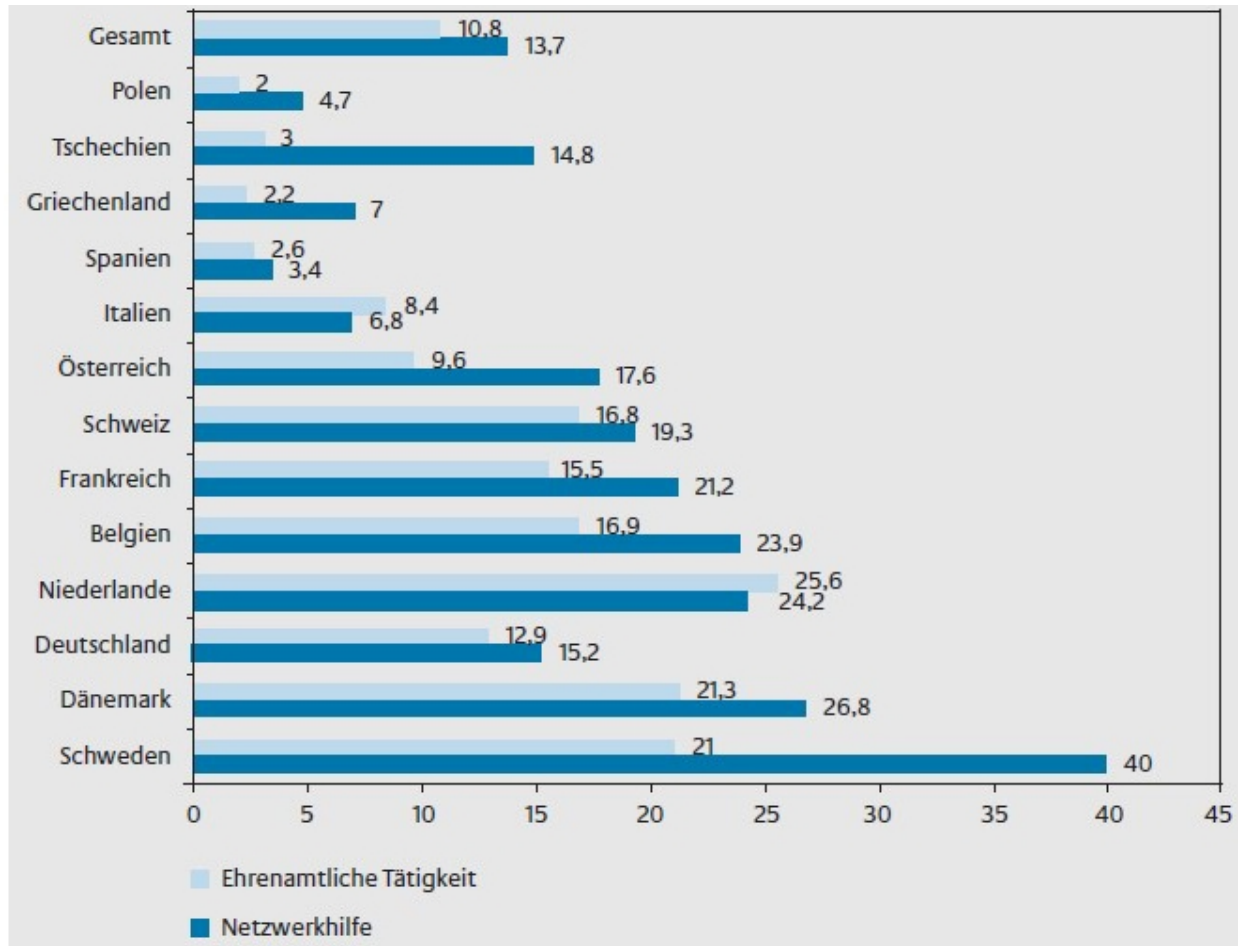

•Prozentualer Anteil an der Bevōlkerung 50 Jahre und älter.

In Bezug auf die Festlegung „älterer Arbeitnehmer“ bei der nationalen Arbeitsmarktpolitik gibt es keine einheitliche Definition, sondern eher eine Konfusion (Eurofound 2018, S. 27 ff.). Aber die international am häufigsten 
verwendete Altersbestimmung ist 55-64 Jahre. In dieser Gruppe herrscht jedoch eine außerordentlich hohe Heterogenität in Bezug auf den Bildungsstand und das Kompetenzniveau, die Gesundheit, den beruflichen Status und die Tätigkeitsfelder.

Dennoch zeigt die international vergleichende Studie nur geringe Unterschiede der nationalen Arbeitsmarktpolitiken hinsichtlich älterer Arbeitnehmer.

In Anbetracht dieser Diskrepanz zwischen der Heterogenität und der relativen Homogenität arbeitsmarktpolitischer Maßnahmen stellt sich die kritische Frage nach der möglichen Ausrichtung an bestimmten Auffassungen zum Verhalten Älterer.

\subsection{THEORIEN DES ERFOLGREICHEN ALTERNS}

Dazu sollen einige herkömmliche Theorien für erfolgreiches Altern (,active aging") kurz behandelt werden (Ertelt, Imsande, Scharpf, Walther 2018, S. $16 \mathrm{ff}$.).

Die „Disengagement-Theorie“ stellt den Rückzug des Menschen ab dem 60. Lebensjahr aus gesellschaftlichen Aktivitäten heraus. Sich zurückzuziehen wird als ein Gewinn an Lebenszufriedenheit gesehen. Kritisch an dieser Theorie ist, dass der Rückzug häufig durch äußere Vorgaben oder Rollenerwartung nahegelegt wird, obgleich offiziell von einem individuell gewollten Ausscheiden aus dem Erwerbsleben ausgegangen wird.

In der „Aktivitätstheorie“ wird die Idee eines intrinsisch motivierten Rückzugs im Sinne der Disengagement-Theorie abgelehnt. Vielmehr wird davon ausgegangen, dass ältere Menschen die gleichen sozialen und psychischen Bedürfnisse haben wie Angehörige des mittleren Erwachsenenalters. Wenn Ältere weniger soziale Kontakte pflegen, hat das ihren Grund in gesellschaftlichen Auffassungen, die den wirklichen Bedürfnissen dieser Menschen entgegen stehen. Daher ist aktiv dagegen anzukämpfen, vor allem bei Eintritt in den Ruhestand. Müssen bisherige Aktivitäten und soziale Kontakte aufgegeben werden, so soll sie der ältere Mensch durch alternative Aktivitäten, etwa soziales Engagement, ersetzen.

Die „Kontinuitätstheorie“ führt diese Gedanken weiter und betont, dass im Alter möglichst die bisherigen aktiven Grundlinien des Lebens beibehalten werden sollten. Dabei beziehen sich die ,innere Kontinuität“ auf die Wahrung der persönlichen Identität (Eigenschaften, Emotionen, Präferenzen, Interessen, Ideen) und die „äußere Kontinuität“ auf die Konstanz der Reprä- 
sentanz im sozialen Umwelt. Hierbei spielt die Interaktion mit vertrauten Personen die zentrale Rolle.

Ein weiterer theoretischer Ansatz geht von der Kompensation altersbedingter Verluste aus. Nach dem sogenannten SOK-Modell kann es dem älteren Menschen gelingen, durch Anpassungsprozesse der Selektion (S), der Optimierung (O) und der Kompensation (K) möglichst lange sein Aktivitätsniveaus, ein positives Selbstbild und eine hohe subjektive Lebensqualität aufrechtzuerhalten. Selektion bedeutet feste Zielsetzungen für die Bündelung vorhandener Ressourcen zu haben. Optimierung bezieht sich auf den Erwerb neuer Fertigkeiten und Kompensation definiert den Einsatz von Mitteln gegen den Verlust von Handlungsmöglichkeiten durch Aktivierung individueller Ressourcen und sozialer Netzwerke (Baltes, Baltes 1990).

Die folgende Tabelle 2 zeigt die dargestellten theoretischen Ansätze in einer Synopse (Ertelt, Imsande, Scharpf, Walther 2018, S. 19):

Tab. 2: Überblick ausgewählter Theorien des erfolgreichen Alterns

\begin{tabular}{|c|c|c|c|}
\hline Theorie & Grundannahme & Gestaltung Alltag & $\begin{array}{l}\text { Bewertung: } \\
\text { Pro \& Contra }\end{array}$ \\
\hline $\begin{array}{l}\text { Aktivitäts- } \\
\text { theorie }\end{array}$ & $\begin{array}{l}\text { Der alternde Mensch } \\
\text { will sozial aktiv sein } \\
\text { und strebt soziales } \\
\text { Teilhaben an. }\end{array}$ & $\begin{array}{l}\text { Der Mensch ist nur } \\
\text { glücklich und } \\
\text { zufrieden, wenn er } \\
\text { aktiv ist, etwas lei- } \\
\text { sten kann, } \\
\text { Aufgaben hat und } \\
\text { gebraucht wird. } \\
\text { Daher sucht er } \\
\text { nach } \\
\text { entsprechenden } \\
\text { Aufgaben. }\end{array}$ & $\begin{array}{l}\text { + Soziale Integration / } \\
\text { Interaktionen als } \\
\text { wichtiger Faktor für } \\
\text { Lebensqualität } \\
\text { - Notwendigkeit einer } \\
\text { Aktivität kann auch } \\
\text { überfordernd wirken } \\
\text { - Aktivität führt nicht per } \\
\text { se zu erhöhter } \\
\text { Lebensqualität (abhängig } \\
\text { von Aktivität und } \\
\text { Persönlichkeit) }\end{array}$ \\
\hline $\begin{array}{l}\text { Disengage- } \\
\text { ment-Theorie }\end{array}$ & $\begin{array}{l}\text { Der alternde Mensch } \\
\text { löst sich zunehmend } \\
\text { aus den bisherigen } \\
\text { Rollen. }\end{array}$ & $\begin{array}{l}\text { Passive, häusliche } \\
\text { Menschen altern } \\
\text { bei Ermöglichung } \\
\text { des Rückzugs } \\
\text { zufriedener. } \\
\text { Spannungen } \\
\text { ergeben sich bei } \\
\text { Inkongruenz, wenn } \\
\text { sich die } \\
\text { Gesellschaft, z.B. } \\
\text { die Angehörigen, }\end{array}$ & $\begin{array}{l}\text { + Überforderung } \\
\text { unwahrscheinlich } \\
\text { - Möglicher Vorwand zum } \\
\text { (gesellschaftlichen) } \\
\text { Ausschluss Mischung aus } \\
\text { Pausen und Aktivitäten } \\
\text { sinnvoll } \\
\text { - Sozialer Rückzug (z.B. } \\
\text { Ausbleiben von Besuch) } \\
\text { kann zur Vereinsamung } \\
\text { führen }\end{array}$ \\
\hline
\end{tabular}




\begin{tabular}{|c|c|c|c|}
\hline & & $\begin{array}{l}\text { mit dem Wunsch } \\
\text { nach vermehrten } \\
\text { Kontakten } \\
\text { durchsetzen. }\end{array}$ & \\
\hline $\begin{array}{l}\text { Kontinui- } \\
\text { tätstheorie }\end{array}$ & $\begin{array}{l}\text { Entsprechend } \\
\text { vergangener innerer } \\
\text { und äußerer Zustände } \\
\text { sollte auch der spätere } \\
\text { Lebensalltag gestaltet } \\
\text { sein, um eine hohe } \\
\text { Lebenszufriedenheit } \\
\text { zu gewährleisten. }\end{array}$ & $\begin{array}{l}\text { Häusliche } \\
\text { Menschen } \\
\text { tendieren zum } \\
\text { Rückzug und } \\
\text { empfinden diesen } \\
\text { als Erleichterung. } \\
\text { Aktive Menschen } \\
\text { benötigen auch im } \\
\text { Alter viele soziale } \\
\text { Kontakte. }\end{array}$ & $\begin{array}{l}\text { + Berücksichtigung des } \\
\text { bisherigen Lebenswegs } \\
\text { - Veränderungen werden } \\
\text { per se als etwas Negatives } \\
\text { gesehen können dabei } \\
\text { positiv sein wenn selbst } \\
\text { gewählt/gut vorbereitet) }\end{array}$ \\
\hline $\begin{array}{l}\text { Kompensations } \\
\text { theorie (SOK } \\
\text { Modell) }\end{array}$ & $\begin{array}{l}\text { Altersbedingte } \\
\text { Defizite können durch } \\
\text { Anpassungsprozesse } \\
\text { ausgeglichen werden. } \\
\text { Gelingt dies, sind ein } \\
\text { stabiles } \\
\text { Funktionsniveau, ein } \\
\text { positives Selbstbild } \\
\text { und ein subjektives } \\
\text { Wohlbefinden } \\
\text { möglich. }\end{array}$ & $\begin{array}{l}\text { Reduzierung der } \\
\text { Aktivitäten, } \\
\text { Verstärktes Üben } \\
\text { dieser Aktivitäten, } \\
\text { Kompensation } \\
\text { durch z.B. } \\
\text { Inanspruchnahme } \\
\text { von Hilfsmitteln }\end{array}$ & $\begin{array}{l}+ \text { Abbauprozesse als } \\
\text { kompensierbar } \\
\text { - Angestrebte Ziele } \\
\text { müssen durch Selektion }+ \\
\text { Kompensation erreicht } \\
\text { werden können, sonst } \\
\text { Frustration } \rightarrow \text { erfordert } \\
\text { gute Selbsteinschätzung / } \\
\text { Reflexion }\end{array}$ \\
\hline
\end{tabular}

Kritisch für die Beratung ist anzumerken, dass solche modellhaften Darstellungen die Realität zwar nur bedingt wiedergeben, aber doch zur Bildung beraterischer Hypothesen anregen können.

Ein wichtiger Bereich muss dabei die Lebensqualität im Sinne der individuellen Lebenszufriedenheit sein. Und diese hängt nicht ausschließlich mit Beruf und Arbeit zusammen, sondern auch mit einer Reihe anderer Aktivitäten, nämlich (Ertelt, Frey 2014, S. 303):

- Stärkung der Verantwortung für die eigene Lebensgestaltung und Verringerung kollektiver Zuständigkeiten.

- Verantwortung für die Entwicklung des eigenen Humankapitals und das der Nachkommen.

- Politisches Engagement in Bezug auf die Erhaltung demokratischer Strukturen im Sinne der Kontrolle über politische Entscheidungsprozesse. 
- Pflege des persönlichen Umfeldes, in welchem in allen Kulturen $\mathrm{Zu}$ friedenheit und Vertrauen ihre Basis haben.

- Arbeiten bis ins höhere Alter, auch wenn es nicht immer im ursprünglichen Beruf ist.

\section{3. ÄLTERE AUF DEM ARBEITSMARKT}

Ältere Arbeitnehmer in Beschäftigung zu halten oder wieder in den Arbeitsmarkt zu integrieren sind wesentliche Aufgaben vor allem der aktiven Arbeitsmarktpolitik. Dazu wurde von Mandl et al. (2018) eine vergleichende Studie für die EU vorgelegt.

Die folgende Abbildung 5 gibt einen Überblick über mögliche Maßnahmen.

Abb. 5 : Instrumente zur (Re)Integration älterer Arbeitnehmer in den Arbeitsmarkt (Mandl et al. 2018, S. 30)

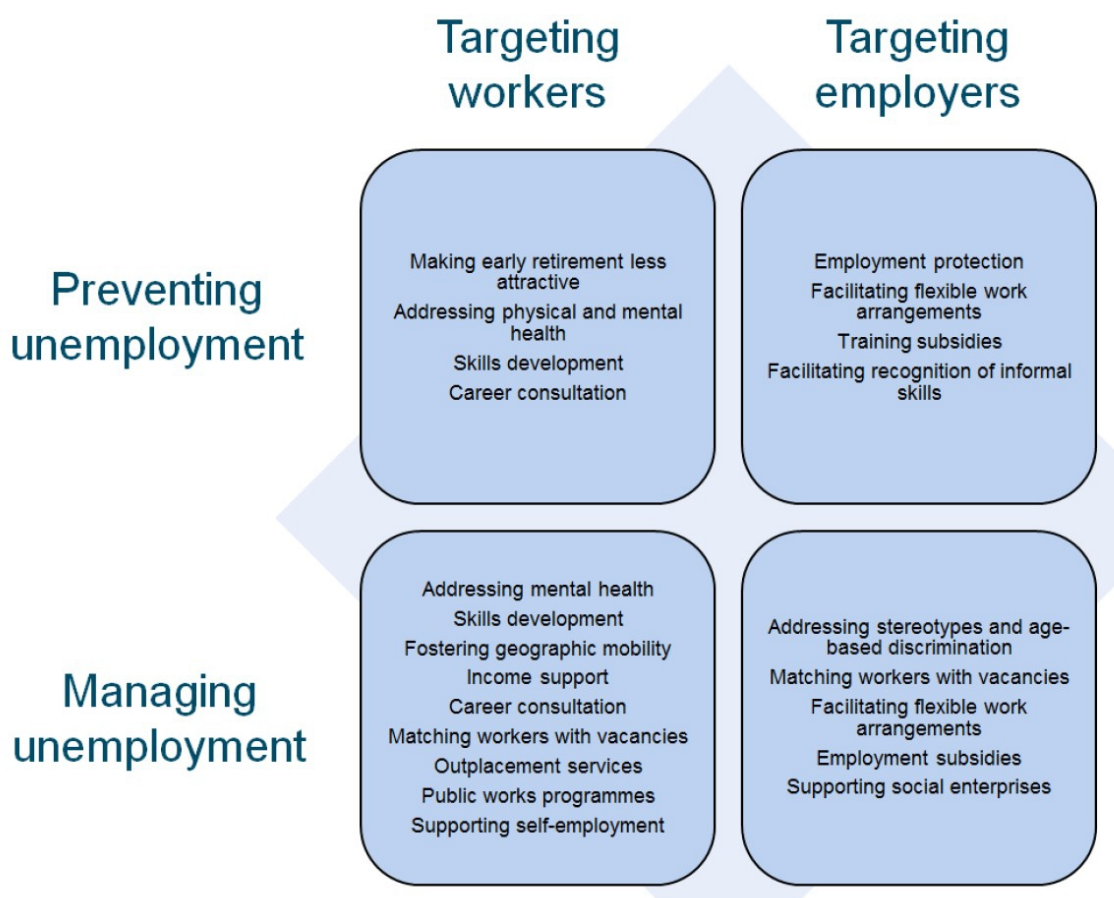


Festgestellt wurde auch, dass die Gesamtpolitik und die Strategien in den EU-Ländern sich eher antizipatorisch auf die Verlängerung der Erwerbstätigkeit richten. Demgegenüber zielen die operationalen Instrumente stärker auf Maßnahmen für ältere Arbeitslose, und nur zu einem Drittel auf die Prävention.

Beratungs- und Vermittlungsdienstleistungen für ältere Arbeitssuchende werden in der Regel von den Public Employment Services (PES) angeboten. Dabei werden individuelle Handlungspläne auf der Basis von Profiling, Berufsberatung und Job Search-Methoden in den Vordergrund gestellt.

Doch haben die vielfältigen Aktivitäten nicht alle die gleiche Effektivität. Die folgende Aufstellung zeigt die verschiedenen Maßnahmen in ihrem Erfolg (Mandl et al. 2018, S. 48):

\section{Positive Effekte}

- Maßnahmen gegen Altersdiskriminierung und Stereotype, aber es bedarf eines langfristigen Ansatzes zum wirklichen kulturellen Wandel.

- Reduzierung der Arbeitsphase in der letzten Erwerbsphase zur nachhaltigen Sicherung des Arbeitslebens in bestimmten Berufen (zum Beispiel: Berufe mit hoher körperlicher Belastung oder gesundheitlichen Gefahren).

- Frühe Intervention und individuelles Fallmanagement zur Bewältigung gesundheitlicher Probleme.

- Längerfristig angelegtes Lernen am Arbeitsplatz.

- Reduzierung von Anreizen für ältere Arbeitnehmer, früher in Ruhestand zu gehen oder in Arbeitslosigkeit zu verharren.

- Regelmäßige intensive Einzelberatungen für ältere Arbeitnehmer mit holistischen Beratungsansätzen (Profiling, Identifikation von Ausbildungsbedarfen und Vermittlungsunterstützung).

- Networking und Erfahrungsaustausch zwischen älteren Arbeitnehmern.

- Umfassende Unterstützungspakete für Praxiserfahrungen und theoretische Ausbildungseinheiten, für Beschäftigungsförderung und Beratung (inklusive Networking).

- Rechtzeitige Intervention möglichst schon ab der ersten Meldung von Beschäftigungsproblemen.

\section{Eher ambivalente Effekte}

- Kündigungsvorschriften für ältere Mitarbeiter können durchaus Probleme für die Neueinstellung von arbeitslosen Älteren nach sich ziehen.

- Subventionierte Arbeit für Ältere kann auch negative Signalwirkungen haben. 
- Staatliche Förderprogramme haben kurzfristig positive Integrationswirkungen auf den Arbeitsmarkt, scheinen jedoch keine nachhaltigen Lösungen zu bieten.

- Gesetzlich verordnete Aktivitäten zur Arbeitssuche haben einige positive Wirkungen für die arbeitsmarktliche Integration älterer Arbeitsloser, doch nach einiger Zeit bilden sich alternative Formen der Entlassung hieraus.

\section{Eindeutig negative Effekte}

- Isolierte Bildungsmaßnahmen, besonders in Form von theoretischen Kurzzeittrainings für gering qualifizierte ältere Arbeitslose.

- Allgemeine Beratung, die sich nicht an der spezifischen Lage älterer Arbeitnehmer orientiert.

Interessant sind hier die Sichtweisen von Betrieben in Deutschland hinsichtlich der besonderen Bedingungen für die Einstellung von Personen ab 50 Jahren (Czepek, Moczall 2017). Bei den Gründen für eine Einstellung stehen „spezielle Kenntnisse und Fertigkeiten“ an weitaus erster Stelle, gefolgt von „nur ohne oder mit nur geringen gesundheitlichen Einschränkungen“. Im Abstand folgen „nicht nach langer Arbeitslosigkeit“, „nur mit Lohnkostenzuschüssen“.

Sinnvolle Maßnahmen zur Verbesserung der Beschäftigungschancen von ab 50-jährigen aus Sicht der Betriebe sind vor allem

1) Lohnkostenzuschüsse an Arbeitgeber bei der Einstellung

2) Staatliche Zuschüsse für Weiterbildung

3) Flexibler Renteneintritt (vom Staat bezahlt)

4) Leistungsbezogene Entlohnung

5) Flexibler Renteneintritt (vom Arbeitgeber bezahlt

6) Lockerung des Kündigungsschutzes

7) Lohnkostenzuschüsse an Arbeitgeber dauerhaft

8) Arbeitgeber gibt Arbeitszeit für Weiterbildung

9) Betriebliches GesundheitsManagement

10) Weiterbildungsmaßnahmen vom Arbeitgeber.

Positiv zu bewerten ist, dass Zugeständnisse in Bezug auf den Lohn bei den Betrieben bei der Neueinstellung kaum eine Rolle spielen.

Insgesamt berichten die Betriebe, dass sie mit den neu eingestellten älteren Arbeitnehmern überwiegend zufrieden sind. Doch lässt sich nicht übersehen, dass bei den Empfehlungen für sinnvolle Maßnahmen zur Verbesserung der Beschäftigungschancen Älterer finanzielle Leistungen des Staats im Vordergrund stehen. 
Aber bei der tatsächlichen Einstellung von Arbeitskräften ab 50 Jahren spielen doch die Berufserfahrung und die Bereitschaft zur Teilzeitarbeit eine wesentliche Rolle.

Eine weitere Untersuchung in Deutschland zeigt, wie die Fachkräfte für Arbeitsvermittlung der öffentlichen Arbeitsverwaltung (Bundesagentur für Arbeit / BA) die Chancen zur beruflichen Eingliederung Älterer beurteilen (Homrighausen, Wolf 2018).

Danach sind nahezu alle Arbeitsvermittler der Meinung, dass Ältere spezifische Hemmnisse haben, die eine neue Beschäftigung erschweren. Dazu gehören

1) Gesundheitliche Einschränkungen

2) $\mathrm{Zu}$ geringe oder veraltete Computerkenntnisse

3) Verschiedene Vorbehalte bei den Betrieben gegenüber Älteren

4) Eingeschränkte geografische Mobilität (besonders bei Frauen)

5) Einschränkungen durch familiäre Verpflichtungen (besonders bei Frauen)

6) $\mathrm{Zu}$ hohe Lohnansprüche

7) Zu geringe Fremdsprachenkenntnisse

8) Eingeschränkte Bildungsmotivation

9) Veraltete oder fehlende formale berufliche Qualifikation.

Als wichtige Instrumente für eine effektive Unterstützung sehen die Vermittler

a) Intensive persönliche Beratung

b) Finanzielle Unterstützung der Betriebe bei der Integration Älterer

c) Beratung der Betriebe zum Thema Älterer.

Empfohlen wird auch - zumindest für über 55-Jährige - ein Lohnzuschuss direkt an die Betriebe.

Eine vergleichende Studie über die Herausforderungen an ältere Arbeitnehmer in Europa kommt zu dem Schluss, dass trotz zunehmender Aufmerksamkeit auf aktives Altern und zunehmender Beschäftigungsquoten Einflussfaktoren existieren, die sich negativ auf eine Verlängerung der Erwerbstätigkeit auswirken (CEDEFOP 2015, S. 15).

$\mathrm{Zu}$ diesen Faktoren gehören

- Institutionelle Herausforderungen, die sich auf die gesellschaftliche Ebene beziehen (,Systemic barriers")

- Situationsbezogene Herausforderungen, die sich auf organisatorischer Ebene ergeben („Situational barriers“) anlagebedingte (dispositive) Heraus- 
forderungen, die sich auf die individuelle Ebene beziehen („Dispositional barrriers").

Die folgende Tabelle zeigt eine Synopse der Einflussfaktoren.

Tab. 3: Overview of challenges to keeping older workers employed

(CEDEFOP 2015, S. 16)

\begin{tabular}{|c|c|c|}
\hline Systemic barriers & Situational barriers & Dispositional barriers \\
\hline Eligible pension age & $\begin{array}{l}\text { Social attitudes in the } \\
\text { workplace and expectations } \\
\text { towards older workers }\end{array}$ & $\begin{array}{l}\text { The generally negative } \\
\text { image of older workers } \\
\text { could form a self-fulfilling } \\
\text { prophecy. }\left({ }^{\text {a }}\right)\end{array}$ \\
\hline $\begin{array}{l}\text { Legislation and tax/benefit } \\
\text { structures, for example with } \\
\text { regard to early retirement }\end{array}$ & $\begin{array}{l}\text { Lack of age aware human } \\
\text { resource policies and } \\
\text { guidance activities }\end{array}$ & $\begin{array}{l}\text { Loss of confidence and } \\
\text { self-esteem and } \\
\text { unwillingness to learn or } \\
\text { tiredness }\left({ }^{b}\right)\left({ }^{c}\right)\end{array}$ \\
\hline $\begin{array}{l}\text { Lack of access to active } \\
\text { labour market services after } \\
\text { retirement age. }\end{array}$ & $\begin{array}{l}\text { Companies' cost-benefit } \\
\text { analyses of investments in } \\
\text { training older workers }\left({ }^{\mathrm{a}}\right)\end{array}$ & $\begin{array}{l}\text { Lack of vocational } \\
\text { qualifications and practical } \\
\text { skills (outdated skills) }\left(^{d}\right)\end{array}$ \\
\hline $\begin{array}{l}\text { The economic situation and } \\
\text { demographic developments }\end{array}$ & $\begin{array}{l}\text { Family circumstances (caring } \\
\text { responsibilities for senior } \\
\text { family members) }\left(^{d}\right)\end{array}$ & $\begin{array}{l}\text { Internal urges to exchange } \\
\text { work for leisure or a life } \\
\text { away from formal work } \\
\text { (voluntary) }\end{array}$ \\
\hline $\begin{array}{l}\text { Society's } \\
\text { view/perception/prejudices } \\
\text { towards older workers }\end{array}$ & $\begin{array}{l}\text { Working conditions and work } \\
\text { structures which do not } \\
\text { directly support further } \\
\text { learning }\left(^{c}\right)\end{array}$ & $\begin{array}{l}\text { The ability to manage and } \\
\text { cope with change in later } \\
\text { life }\left({ }^{a}\right)\end{array}$ \\
\hline $\begin{array}{l}\text { Lack of incentive for } \\
\text { companies to deploy active } \\
\text { age management policies }\end{array}$ & $\begin{array}{l}\text { Changing and emerging } \\
\text { career development models } \\
\text { and flexibilisation of the } \\
\text { labour market }\end{array}$ & $\begin{array}{l}\text { Lack of understanding of } \\
\text { how the labour market } \\
\text { operates }\left({ }^{\mathrm{e}}\right)\end{array}$ \\
\hline \multirow[t]{2}{*}{$\begin{array}{l}\text { Lack of guidance and lifelong } \\
\text { learning opportunities }\end{array}$} & $\begin{array}{l}\text { Older workers' competences } \\
\text { concentrate increasingly on a } \\
\left.\text { specialised field of work ( }{ }^{c}\right)\end{array}$ & $\begin{array}{l}\text { Financial situation of older } \\
\text { workers (driver as well as } \\
\text { hampering factor) }\end{array}$ \\
\hline & $\begin{array}{l}\text { Opportunities to keep up to } \\
\text { date with technological } \\
\text { changes, lack of information, } \\
\text { reduced social networks }\left({ }^{b}\right)\end{array}$ & $\begin{array}{l}\text { Physical and mental health } \\
\text { problems }\left({ }^{c}\right)\end{array}$ \\
\hline
\end{tabular}

\section{ARBEITSBEZOGENE ERWARTUNGEN ÄLTERER ARBEITNEHMER}

Im Rahmen des ERASMUS+ - Projekts „INCounseling 50+“, unter der Leitung der HdBA, wurde von März-Mai 2017 in den Partnerländern Deutschland, Kroatien und Polen eine empirische Studie ( $n=411 ; 207$ Frauen 
und 214 Männer) anhand einer schriftlichen Befragung von drei Gruppen durchgeführt:

- Beschäftigte 50+ (Employees 50+)

- Arbeitslose / Arbeitssuchende 50+

- Arbeitnehmer im Übergang von der Erwerbstätigkeit in den Ruhestand (Employees in transition).

\subsection{MOTIVATION, BEDÜRFNISSE UND ERWARTUNGEN IM ARBEITSLEBEN}

\section{Beschäftigte 50+}

a) Faktoren, die motivieren:

1. Gute Arbeitsatmosphäre

2. Respekt und Anerkennung gegenüber der geleisteten Arbeit

3. Sicherheit, dass man die Arbeit behält (Beschäftigungssicherheit

b) Bedürfnisse und Erwartungen:

1. Gute und sichere Arbeitsbedingungen (incl. finanzielle Sicherheit)

2. Gute Arbeitsatmosphäre

3. Beschäftigungssicherheit

1. Sicherheit/Stabilität der Beschäftigung

2. Respekt und Anerkennung der Beschäftigung

3. Finanzielle Faktoren.

\subsection{PLÄNE BIS ZUM RUHESTAND UND DANACH}

\section{Beschäftigte 50+}

a) Pläne: Wunsch nach Aufrechterhaltung der gegenwärtigen Position und Arbeitsbedingungen im gleichen Betrieb

b) Die Pläne für den Ruhestand sind verbunden mit freier Zeit für Familie und Hobby

c) Emotionen in Bezug auf den Ruhestand: Freude und Erleichterung

\subsection{ERWARTUNGEN UND WÜNSCHE AN BERATUNGSDIENSTLEISTUNGEN}

\section{Beschäftigte 50+}

Die Beratung wird grundsätzlich als positiv beurteilt, sie sollte als Einzelberatung möglichst arbeitsplatznah stattfinden, wenn möglich als persönliche Treffen alle halbe Jahre vor der Transition in den Ruhestand. 
Primär sollten folgende Themen besprochen werden:

- Persönliche Gesundheitsvorsorge

- Finanzfragen

- Rechtliche Beratung

- Freizeitgestaltung

- Weitere Beschäftigung oder berufliche Entwicklung

- Finanzfragen

- Freizeitgestaltung

- Weitere Beschäftigung oder berufliche Entwicklung

Bei den Wünschen fällt die große Ähnlichkeit zwischen den befragten Gruppen auf. Besonders zu beachten ist der durchgängige Wunsch nach möglichst arbeitsplatznaher Einzelberatung.

Für das Beratungsmarketing bedeutet dies, die Distribution möglichst mit dem Betrieb zu verzahnen. Eine betriebsexterne Beratung erscheint dagegen wenig attraktiv zu sein.

\subsection{MEINUNG DER HR-SPEZIALISTEN ÜBER BESCHÄFTIGTE 50+}

Bei der Untersuchung im Rahmen von „INCounselling 50+“ erfolgte in einem zweiten Schritt eine qualitative Studie bei HR-Professionals $(n=21)$ anhand halbstandardisierter (semi-structured) Interviews, deren Ergebnisse im Folgenden zusammengefasst werden.

Überwiegend waren die HR-Spezialisten der Meinung, dass die älteren Mitarbeiter für den Betrieb wichtig sind. Im einzelnen wurden folgende Stärken genannt:

- Erfahrung und Kenntnisse

- Loyalität gegenüber dem Arbeitgeber

- Stabilität und Ruhe

- Verantwortungsbewusstsein

- Gute Arbeitstugenden, wie Pünktlichkeit, Sorgfalt, Respekt, Selbstvertrauen

- Arbeits- und Lernmotivation

- Kollegialität

- Gute Aufgabenerfüllung.

Doch auch Schwächen wurden auch genannt:

- Vor allem mangelnde Flexibilität

- Verharren auf bisherigen Verfahrensweisen

- Langsameres Arbeiten 
- Ungenügendes Gesundheitsbewusstsein

- Kompetenzmängel hinsichtlich IT-Anwendungen

- Einschränkungen bei Arbeiten unter Stress und Zeitdruck.

Als notwendig erachtet wurden deshalb Anpassungsmaßnahmen am Arbeitsplatz, spezielle Gesundheitsprogramme, geänderte Arbeitsformen (z.B. Heimarbeit, Job Rotation) und Arbeitszeiten. Die wichtigsten Motivatoren für Ältere seien Sicherheit des Arbeitsplatzes, finanzielle Anreize, Respekt und Anerkennung.

Die HR-Spezialisten sehen Probleme durch den Verlust von Erfahrungswissen beim Übertritt in den Ruhestand und fragen nach Möglichkeiten, Ältere möglichst lange im Betrieb zu halten.

\section{AUFGABEN DES AGE-MANAGEMENTS ALS GRUNDLAGE FÜR BERATUNG}

Im Folgenden werden zuerst die Methoden des Age-Managements aus betrieblicher Sicht beschrieben.

Danach gehen wir auf die Phase der Vorbereitung auf den Ruhestand eher aus individueller Sicht ein.

Eine aktuelle Studie in Deutschland (Bellmann, Dummert, Leber 2018) zeigt, dass trotz steigender Beschäftigung Älterer spezifische Personalentwicklungsmaßnahmen für diese Zielgruppe nach wie vor nur eine untergeordnete Rolle spielen. Ein Grund dafür ist, dass die Betriebe bei ihren Personalmaßnahmen gar nicht zwischen Altersgruppen differenzieren. Allerdings ergaben sich hierbei doch Unterschiede nach der Betriebsgröße, denn größere Betriebe bieten signifikant häufiger spezielle Maßnahmen für ältere Beschäftigte $(50+)$ an als kleinere Betriebe. In letzteren findet man eher Maßnahmen, die sich auf einzelne Mitarbeiter je nach Bedarf beziehen. Die Autoren ziehen folgendes Fazit: „Im Sinne einer vorausschauenden Personalpolitik kommt es dabei auch wesentlich darauf an, bereits frühzeitig in die Beschäftigungsfähigkeit des Personals zu investieren und nicht erst im Alter damit zu beginnen."

In einer empirisch-kasuistischen Studie über das Marketing für berufsbezogene Weiterbildung Älterer in Betrieben kommt Braun (2018) zu dem Ergebnis, dass Zeitmangel als eines der größten Hindernisse für Weiterbildung 
besonders in den KMU betrachtet wird. Außerdem verfügen diese oft über keine eigene Stelle für die Personalentwicklung.

Insgesamt spielt das Alter offenbar keine wesentliche Rolle, selbst beim Thema Digitalisierung und neue Technologien sind die Älteren nicht im Nachteil. Bei der Weiterbildung werden altersgerechte Gruppen bevorzugt. Alle befragten Betriebe betonten die Bedeutung, der Generation 50+ mit dem nötigen Respekt zu begegnen. Auch wird das Wort „älter“ im Betrieb nicht gerne benutzt, man ersetzt es durch „erfahrenere Mitarbeiter“ oder „Generation 50+“, weil in den meisten Köpfen noch immer ein negatives Altersbild von körperlicher und geistiger Einschränkung vorherrsche.

Auch wenn nicht in jedem Betrieb/Unternehmen ein professionelles AgeManagement existiert, so wird doch die Notwendigkeit dazu allgemein anerkannt. Hafkesbrink und Krause (2017, S. 41 ff.) benennen dazu die folgenden Handlungsfelder und Leitlinien zur Prüfung der Umsetzung:

Handlungsfeld Unternehmenskultur

- Das Unternehmensleitbild benennt „Diversity“ der Mitarbeiter als Prinzip.

- Es herrscht eine Kultur des Respekts und der Wertschätzung.

- Es bestehen keine Vorurteile nach Altersverschiedenartigkeit.

- Alle Mitarbeiter identifizieren sich mit dem Leitbild des Unternehmens.

- Alle Mitarbeitergruppen werden sensibilisiert für die Folgen des demografischen Wandels auf das Unternehmen und jeden Einzelnen.

Handlungsfeld Führung

- Alle Führungskräfte sind auf generationsbergreifende Führungskompetenzen vorbereitet.

- Die Führungskräfte achten auf eine Altersbalance bei der Beteiligung an Entscheidungsprozessen.

- Die Führungskräfte achten auf die verantwortliche Beteiligung der Mitarbeiter an Veränderungsprozessen im Betrieb.

- Die Führungskräfte fördern den Dialog von jüngeren und älteren Mitarbeitern sowie die Zusammenarbeit „mixed teams“.

Handlungsfeld Personalmanagement

- Die Personalplanung ist demografie-bezogen mit einem Zeithorizont von 5 bis 10 Jahren.

- Unser Unternehmen praktiziert eine rechtzeitige und systematische Nachfolgeplanung. 
- Das Unternehmen bezieht bei der Personalrekrutierung ein breites Spektrum von Zielgruppen mit ein.

- Das Unternehmen bietet allen Mitarbeitern eine individuelle Laufbahnplanung mit alternativen Entwicklungsoptionen.

- Das Unternehmen praktiziert eine Strategie des lebensbegleitenden Lernens für alle Mitarbeitergruppen.

Handlungsfeld Arbeitsorganisation

- Die Arbeitsabläufe sind so gestaltet, dass die Belastungen für die Mitarbeiter möglichst reduziert werden.

- In unserem Unternehmen sorgen Tätigkeitswechsel und Arbeitsplatzwechsel für eine Erhöhung der Flexibilität.

- Die Arbeitszeitgestaltung harmonisiert die Anforderungen aus dem Arbeitsprozess und den individuellen Möglichkeiten und Wünschen der Mitarbeiter.

- Wir fördern die altersgerechte Gestaltung der Arbeitsplätze.

- Unser Unternehmen ist der Familienfreundlichkeit verpflichtet.

Handlungsfeld Gesundheit

- Wir führen regelmäßige Analysen von Belastungen am Arbeitsplatz und Fehlzeiten durch.

- Im Unternehmen werden Mitarbeiter für eine präventive Gesundheitsförderung sensibilisiert.

- Wir achten auf eine angemessene Work-Life-Balance bei unseren Mitarbeitern.

- Für Mitarbeiter nach längeren Phasen der Arbeitsunfähigkeit steht ein aktives betriebliches Eingliederungsmanagement zu Verfügung.

- An Arbeitsplätzen mit belastenden Umgebungsbedingungen werden altersbedingte Besonderheiten berücksichtigt.

Handlungsfeld Innovation und Wissenstransfer

- Wir haben ein beteiligungsorientiertes Innovationsmanagement, in das wir das Erfahrungswissen der älteren Mitarbeiter systematisch einbeziehen.

- Das Unternehmen identifiziert die Personen, die wettbewerbswichtiges Wissen besitzen und machen sie zu Teilnehmern des zentralen WissensManagements.

- Es werden Strukturen aufgebaut, die den Austausch von Wissen und Erfahrungen von Jüngeren und Älteren systematisch fördern.

- Unser Unternehmen verbindet das Wissensmanagement mit der Unternehmenssteuerung, Organisationsentwicklung und Innovationsstrategie. 
Lässt man im Rahmen des Management-Audit die Aspekte der Handlungsfelder auf einer Rating-Skala (z.B. 6er Skala) von Führungskräften im Zeitablauf (time-line) bewerten, kann das Age-Management systematisch optimiert werden. Dazu lassen sich die jeweiligen Ausprägungen als SummenScores in einem Netz-Diagramm abbilden, wie folgendes Beispiel zeigt (Hafkesbrink, Krause 2017, S. 41):

Abb. 6: Beispiel eines Netz-Diagramms zum Age-Management

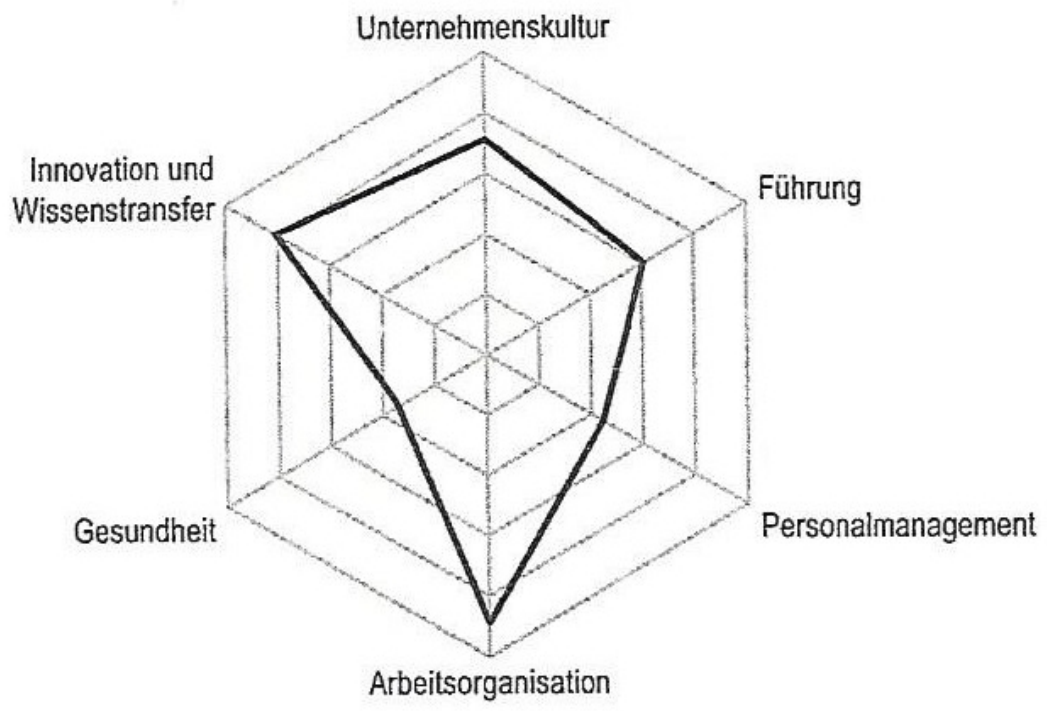

In diesem Beispiel zeigt das Audit, dass der Betrieb bereits gut vorangekommen ist in Bezug auf die altersgerechte „Arbeitsorganisation“ sowie den Bereichen „Innovation und Wissenstransfer“".

Doch obgleich der demografische Wandel unmittelbare Auswirkungen auf den Unternehmenserfolg hat, ist ein systematisches Age-Management in vielen Betrieben noch nicht selbstverständlich. Die „Towers Watson Studie“ (2014) gibt Unternehmen fünf Empfehlungen, die Folgen demografischer Veränderungen erfolgreich zu bewältigen:

1. Abschätzung des zukünftigen Personalbedarfs durch eine Personalbestandsanalyse und die Projektion in Bezug auf die Rekrutierung bestimmter Zielgruppen, einschließlich Älterer. 
2. Verstärkung des „Employer Branding“ mit Blick auf die Bindung vorhandener und die Rekrutierung potentieller Arbeitskräfte. Dazu ist das Personalmarketing und das RetentionsManagement gezielt zu verbessern.

3. Erhaltung der Leistungsfähigkeit der Mitarbeiter und eines hohen betrieblichen Engagements, aber Vermeidung von Überbelastung und Förderung einer ausgewogenen Work-Life-Balance.

4. Gestaltung eines flexiblen Übergangs in den Ruhestand, einerseits zur Bindung von Wissens- und Leistungsträgern auch jenseits des 60. Lebensjahres an das Unternehmen und andererseits die Arbeitsbelastung individuell anzupassen. Dazu könnten unternehmensspezifische Lösungen erarbeitet werden, wie etwa die Kombination von betrieblicher Altersversorgung, Zeitwertkonten und Demografiefonds.

5. Einbeziehung der altersspezifischen Veränderung der Nachfrage an Produkten und Dienstleistungen. Denn mit den alternden Mitarbeitern altern oftmals auch die Kunden des unternehmens. Eine rechtzeitige Orientierung an der sogenannten „Silver Economy“ kann den Bestand der Unternehmung auch in Zukunft sichern.

\section{BERATUNG ZUR VORBEREITUNG AUF DEN RUHESTAND}

Sicherlich bedeutet die Phase des Übergangs in den Ruhestand für das Individuum und seine wichtigen Bezugspersonen eine Herausforderung, auf die man nicht immer adäquat vorbereitet ist.

We sometimes forget that retirement and the time immediately preceding it are a part of one's career life; these times are as fraught with potentially anxiety-producing events as are any others. Most of that anxiety for those who have had a work life revolves around the possibility that loss of work will cause serious problems of adjustment (Herr, Cramer, Niles 2004, p. 533).

Doch die verschiedenen wissenschaftlichen Studien geben kein eindeutiges Bild darüber, wie sich der Übergang in den Ruhestand (,transition into retirement") auf das individuelle Wohlbefinden auswirkt. Einerseits gibt es Hinweise darauf, dass sich die unmittelbar nach Eintritt in den Ruhestand entstandene Unzufriedenheit schon nach wenigen Monaten zunehmend in Lebenszufriedenheit und positive Gefühle verwandelt. Andere Studien zei- 
gen, dass das anfänglich große subjektive Wohlbefinden nach einigen Jahren abnimmt (Froidevaux et al. 2017).

Für eine wirksame beraterische Unterstützung des Transitionsprozesses wurden eine Reihe von Typologien entwickelt.

Eine Studie der „Americana Health Care Corporation“ von 1980 gliederte ältere Menschen in drei Gruppen:

1. „Enjoyers“ (27\%), die erfolgreich mit dem Alter umgehen, in der Regel gesund, gebildet und finanziell abgesichert sind.

2. „Survivers“ (53\%), die angemessen mit ihrem Alter umgehen, weitgehend gesund sind, einen, einen mittleren Bildungsabschluss besitzen und ein angemessenes finanzielles Auskommen haben.

3. „Casualties“ (20\%), die nicht sehr erfolgreich die Folgen des Ausscheidens aus dem Erwerbsleben bewältigen, in der Regel weiblich sind, über keine höhere Bildung verfügen, gesundheitliche Einschränkungen haben und nur über ein geringes Einkommen verfügen.

Eine weitere Kategorisierung (Walker, Kimmel, Prices 1981 in: Herr, Cramer, Niles 2004, S. 535) unterteilt in folgende Gruppen:

1. „The Rocking Chair Group“ (44\%): Diese Menschen sind gesund und sehr zufrieden mit ihrem Freundeskreis, finanziellen Status und ihren Aktivitäten.

2. „The Reorganiziser Group“ (24\%): Diese Menschen sind im Alter zwischen 62 und 65 freiwillig in den Ruhestand gegangen, haben eine gehobene Bildung, bessere finanzielle Absicherung und sind gesünder. Sie planen neue Aktivitätsformen und engagieren sich oft ehrenamtlich.

3. „The Holding On Group“ (rund $19 \%$ ): Angehörige dieser Gruppe setzen ihre Erwerbstätigkeit fort. Einige sind unfreiwillig in den Ruhestand gegangen und brauchen den Verdienst. Die meisten von ihnen haben nicht ausreichend über den Ruhestand nachgedacht oder Pläne dafür gemacht. Arbeitsmöglichkeiten haben die absolute Priorität.

4. „The Dissatisfied Group“ (13\%): Diese Menschen arbeiten weder ehrenamtlich noch gegen Lohn und versuchen verzweifelt, aktiv zu bleiben. Einkommen und Gesundheit sind relativ eingeschränkt. Insgesamt fühlen sie sich im Ruhestand nicht sehr wohl.

Trotz einer Reihe von Forschungsarbeiten zum Vorruhestand und Ruhestand bleiben für Herr, Cramer, Niles eine ganze Reihe von Fragen zu folgenden Bereichen der beraterischen Hilfe offen: 
- Bestimmung des Status an physischen und geistigen Fähigkeiten für eine weitere Beschäftigung.

- Angemessene ökonomische und nicht-ökonomische Anerkennungen bei einer Weiterbeschäftigung Älterer.

- Einschätzung der sozial-psychologischen Einflüsse auf die individuelle Meinung zum Ruhestand.

- Einfluss von Persönlichkeitsfaktoren auf die Entscheidung über den Zeitpunkt und die Form, in den Ruhestand zu gehen.

- Wie wirken sich Informationsangebote zur Ruhestandsplanung aus und wieweit kann man überhaupt von einer rationalen Entscheidungsfindung ausgehen?

- Was bewirken unternehmenspolitische Maßnahmen und Arbeitsmarktbedingungen in Bezug auf die individuelle Einstellung zum Ruhestand oder weiteren Erwerbstätigkeit?

Die sicherlich bekannteste Charakterisierung des Verhaltens beim Übergang in den Ruhestand und in der sich anschließenden Lebensphase stammt von Schlossberg (2003). Sie definiert fünf Typen, die sich allerdings auch überlappen können (Froidevaux et al. 2017, S. 31 ff.):

1. Für die „continuers“ bleibt das bisherige Berufsleben der zentrale Bezugspunkt für die eigene Identität und Aktivität. Nur langsam gewöhnen sie sich an die neue Rolle im Ruhestand. Nicht selten arbeiten sie im bisherigen Betrieb weiter (natürlich mit anderer Arbeitszeitgestaltung) oder bleiben als Berater mit ihm verbunden.

2. Den „adventurers“ bietet der Ruhestand die Möglichkeit, ihr Leben neu zu organisieren. Es werden Reisen in andere Länder unternommen, eine andere berufliche Aktivität geplant oder durch Weiterbildung neue Kompetenzen erworben.

3. Die „easy gliders“ betrachten den Ruhestand als Periode der Freiheit, ohne feste Zeitplanung und mit der Möglichkeit, jeden Tag anders zu gestalten, je nach sich bietenden Gelegenheiten.

4. Für die „searchers“ wird der Transitionsprozess in den Ruhestand begleitet von Zweifeln und Ungewissheiten, denn sie haben noch keine Idee, wie es weitergeht oder sie haben unrealisierbare Ideen. Ihr handeln gleicht eher einem Versuch und Irrtum-Konzept.

5. Die „retreaters“ haben zu allem eine negative Wahrnehmung und Gefühle der Konfusion und Nutzlosigkeit. Für einige stellt der Ruhestand das Ende des sinngebenden Arbeitslebens dar. Doch zeigen Untersuchungen, 
dass diese Einstellung oft nur ein vorübergehendes Stadium darstellt, das dann beendet wird, wenn dem Einzelnen konstruktive Möglichkeiten zu Lebensgestaltung geboten werden.

Diese Typologie von Schlossberg (2003) bildete die Basis für den „Transition to Retirement Questionnaire (TQR)“ von Maggiore et al. (2014) zur Einschätzung der individuellen Wahrnehmung der bevorstehenden Transition vom Erwerbsleben in den Ruhestand. Die Studie zur Validierung des TQR liefert wertvolle Hinweise für die Auswahl der richtigen Informationen und Methoden in der Übergangsberatung (Froidevaux et al. 2017, S. 40 ff.).

So sind etwa die wirklichen Profile als „retreater“ oder „searcher“ erst zu erkennen, wenn der Klient schon länger als ein paar Monate im Ruhestand ist. Kann der Berater beim Klienten ein „continuity“ Profil ausmachen, kann man von ein er Spätphase der Berufsentwicklung ausgehen und zum Beispiel empfehlen, den Zeitpinkt der Pensionierung zu verschieben, um so eine Brücke zwischen Erwerbstätigkeit und Ruhestand zu bauen.

Das ,adventurer“ Profil bei Klienten legt nahe, sich beraterisch mit einer neuen Arbeitsumgebung oder Weiterbildung zu beschäftigen.

Für den Typus „easy glider“ könnte es kontraproduktiv sein, wenn der Berater versucht, ihn in Maßnahmen zum Zeit-Management und genauer Lebensplanung einzubinden.

Besonders wichtig sind Beratungsangebote für Menschen, die eine negative Meinung gegenüber dem Ruhestand haben. Drei Zielgruppen erscheinen hiervon besonders betroffen:

- Ältere Erwerbstätige mit niedrigem Einkommen. Hier stehen Fragen der finanziellen Sicherung und der Selbstachtung im Ruhestand im Vordergrund.

- Ältere Erwerbstätige mit gesundheitlichen Einschränkungen und chronischen Erkrankungen. Hier stehen Fragen der Erhaltung der Arbeitsfähigkeit und der Entscheidungsmodalitäten für ein Ausscheiden aus dem Arbeitsprozess im Mittelpunkt.

- Menschen, die im Ruhestand freiwillig oder gezwungenermaßen weiterarbeiten. Die Beratung könnte sich darauf beziehen, wie man seine Träume oder gewonnenen Freiheiten in die Arbeit wenigstens teilweise integriert.

Berufliche Transitionsberatung sollte (1) realistische Erwartungen bezüglich des Ruhestands fördern, (2) Fertigkeiten des Zeit-Managements vermitteln, (3) die Unsicherheiten bezüglich der künftigen sozialen Rollen 
diskutieren und (4) dem Einzelnen helfen, sich stärker mit den Gefühlen und Einstellungen gegenüber der Pensionierung auseinanderzusetzen.

Eine professionelle beraterische Unterstützung bei der Planung des Ruhestandes muss die verschiedenen Einflussgrößen auf das individuelle Verhalten berücksichtigen und kann sich nicht nur von Typologien leiten lassen.

So spielen die sozio-ökonomischen Variablen (Geschlecht, Bildungsniveau, Alter, Einkommen) eine wesentliche Rolle. Studien zeigen, dass Männer bei der Ruhestandsplanung eher an finanzielle Aspekte denken und Frauen eher die interpersonellen Kontakte und die Freizeitgestaltung fokussieren.

Ein höheres Bildungsniveau wirkt sich positiv auf die gesundheitsvorsorge und eine intensivere Finanzplanung aus.

Menschen mit niedrigerem Einkommen scheuen sich offenbar, eine qualifizierte Finanzberatung für den Ruhestand in Anspruch zu nehmen und planen häufiger, weiterhin zu arbeiten.

In Bezug auf die Persönlichkeitsvariablen haben vor allem zwei Faktoren Einfluss auf die Art der Transitionsplanung. Proaktiv eingestellte Menschen zeigen eine stärkere Selbst-Initiative und Zukunftsbezogenheit. Außerdem versuchen sie, ihre Umgebung ihren Bedürfnissen aktiv anzupassen und sind so besser auf den Ruhestand vorbereitet. Dagegen sind Menschen, die eher kurzfristig denken und zeitnahe Belobungen bevorzugen (geringe „deferred gratification") weniger bereit, ihren Ruhestand systematisch zu planen.

Sehr wichtig sind die persönlichen Ziele und positive Einstellungen gegenüber der Transition. Hierin scheint es auch geschlechtsspezifische Unterschiede zu geben, etwa, dass ältere Frauen mit einer positiven Einstellung zum Ruhestand eine sozial angemessene Planung vornehmen.

Hohes Berufsengagement (,work involvement") und hohe Arbeitszufriedenheit wirken sich offenbar positiv auf den Übergangsplanung aus.

Auf der Grundlage ihrer Forschungsergebnisse empfehlen Froidevaux et al. (2017) für die Gestaltung der beruflichen Übergangsberatung den Ansatz des „Life-Designing“ (sensu Savickas et a. 2009; vgl. auch Nota, Rossier (eds.) 2015).

Als grundlegende Rahmenbedingungen für die Life-designing Beratung betonen die Autoren

- die lebensbegleitende Funktion (life-long),

- den holistischen Ansatz, der die Selbst-Konstruktion in allen Rollenbezügen berücksichtigt, nicht nur den der Berufsrolle, 
- die Einbeziehung des gesamten Lebensumfeldes des Klienten (contextual),

- den prägnanten Charakter durch Aufarbeitung der bisherigen Lebensgeschichte des Klienten.

Erst diese Voraussetzungen ermöglichen die Realisierung der zentralen Ziele der Life-designing Beratung:

1. Stärkung der Anpassungsfähigkeit des Klienten an die wechselnden Entwicklungserfordernisse (,Adaptability“).

2. Bezug auf die bisherige und gegenwärtige Biographie und SelbstKonstruktion des Klienten („Narratability“).

3. Stärkung der Eigenaktivität des Klienten, in Bezug auf die Entwicklung neuer Dimensionen der Persönlichkeit, z. B. Selbstwirksamkeitsüberzeugung (,Activity“).

4. Bewusstmachen der individuellen Gestaltungsvorgänge und Entscheidungen im Leben des Klienten, damit diese Zusammenhänge für künftige Selbst-Konstruktionen nutzbar werden (,Intentionality“).

Das Beratungsmodell im Sinne des Life-designing umfasst folgende sechs Schritte:

Schritt 1: Klient und Berater definieren gemeinsam das Problem und identifizieren die Zielerwartungen des Klienten an die Beratung.

Schritt 2: Klient und Berater erforschen, wie der Klient sich gegenwärtig wahrnimmt und in Bezug auf seine wesentlichen Rollenanforderungen verhält.

Schritt 3: Vornehmlich durch narrative Methoden werden dem Klienten Neubestimmungen wichtiger Lebensbezüge ermöglicht und Perspektiven herauskristallisiert.

Schritt 4: Das aktuelle Problem wird in Bezug gesetzt zu den neuen Perspektiven. Gelungen ist dieser Schritt, wenn dem Klienten eine Synthese zwischen Altem und antizipiertem Neuen gelingt.

Schritt 5: Ausarbeitung eines Handlungsplans und Einleitung konkreter Schritte, unter Berücksichtigung der Umfeldbedingungen und Unterstützungsmöglichkeiten des Klienten.

Schritt 6: Nachgehende Betreuung (,follow-up“) in Bezug auf kurzfristige und längerfristige Ergebnisse des Umsetzungsprozesses (Savickas et al. 2011).

Besonders die Anpassungsfähigkeit („Adaptability“) wird in der Konzipierung durch Savickas (2005) in den letzten Jahren zunehmend diskutiert. Danach sind vier Einstellungen des Einzelnen wichtig, damit er sein Selbst- 
konzept in einem Beruf verwirklichen Kann: Zukunftsbezogene Planung („Concern“), berufliche Entschiedenheit („Control“), Zuversicht als berufliche Selbstwirksamkeitserwartung („Confidence“) und Neugierde („Curiosity“).

Die Planung des Ruhestands spielt eine zentrale Rolle bei der Stärkung der individuellen Fähigkeit, die neuen Lebensumstände den eigenen Bedürfnissen anzupassen (Froidevaux et al. 2017, S. 33).

\section{ZUSAMMENFASSUNG}

- Das Demografie- oder Age-Management in Betrieben und Organisationen vollzieht sich in den üblichen Aufgabenbereichen des Human Resource Management jeweils mit spezifischen Herausforderungen

- Zur Feststellung von Qualitätsfortschritten lassen sich für das Audit fünf Handlungsfelder (Unternehmenskultur, Führung, Personalmanagement, Arbeitsorganisation, Gesundheit, Innovation und Wissenstransfer) benennen und operationalisieren

- Für die Beratung in der Transitionsphase zum Ruhestand können Typologien - besonders die von Schlossberg (2003) - zur Charakterisierung von Verhaltensweisen Älterer durchaus hilfreich sein

- Zur methodischen Gestaltung von beruflichen Beratungsprozessen lässt sich der Ansatz des „Life-Designing“ (sensu Savickas et al.) heranziehen, weil er narrativ und systemisch ausgerichtet ist.

- Für die berufliche Beratung von Menschen im Ruhestand gibt es bislang wenig empirische Untersuchungen zu Interessen, Selbsteinschätzung und spezifischen Angebotsformen. Die hier exemplarisch dargestellten Ergebnisse zeigen ausgeprägte berufliche Interessen und positiv eingeschätzte Kompetenzen.

\section{BIBLIOGRAPHIE}

Americana Health Care Corporation (1980). Aging in America. Trials and triumphs. New York: Research and Forecast, Inc.. In: Herr, E.L., Cramer, St.H., Niles, Sp.G. (2004), Career Guidance and Counseling Through The Lifespan - Systematic Approaches. 6th ed. p. 535. Boston et al.: Pearson.

ARBINGER, R. (1999). Entwicklungspsychologie des Jugendalters. Landau: Verlag Empirische Pädagogik. 
Baltes, P.B., Baltes, M.M. (1990): Psychological perspectives on successful aging: The model of selective optimization with compensation. In: P.B. BALTES \& M.M. BALTES (Eds.), Successful aging: Perspectives from the behavioral sciences (s. 1-34). New York: Cambridge UP.

Bellmann, L., Dummert, S., Leber, U. (2018). Konstanz altersgerechter Maßnahmen trotz steigender Beschäftigung Älterer. WSI-Mitteilungen 2018, s. 20-27.

Braun, S. (2018). Marketing für berufsbezogene Weiterbildung Älterer aus betrieblicher SichtEine empirisch-kasuistische Studie. Masterarbeit Universität Mannheim

Bundesministerium für Familie, Senioren, Frauen und Jugend (BMFSFJ) (2011). Monitor Engagement - Wie und wofür engagieren sich ältere Menschen? Ausgabe Nr. 4.

CzepeK, J., Moczall, A. (2017). Neueinstellung Älterer - Betriebe machen meist gute Erfahrungen. Institut für Arbeitsmarkt- und Berufsforschung, IAB-Kurzbericht 8/2017.

ERASMUS-Projekt INCounselling 50+: Project presentation on HdBA page.

ERTELT, B.-J., FreY, A. (2014). Berufsentwicklung und Lebensqualität. In: A. WUDARSKI (ed), Quaestiones de Qualitate Vitae (Auf der Suche nach Lebensqualität) (s. 303-320). Frankfurt/ Oder, Osnabrück, AJD Częstochowa.

Ertelt, B.-J., Imsande, A., Scharpf, M. \& Walther, Th. (2018). Arbeit, Leben, Teilhabe ab 55 - Trainingsmodule für Beratende. Bielefeld: Media GmbH \& Co. KG.

European Centre for the Development of Vocational Training (CEDEFOP) (2015). Increasing the value of Age - Guidance in employers' Age Management strategies. Research Paper Nr. 44. Luxembourg: Public Office of the European Union.

EUROPEAN FOUNDATION FOR THE IMPROVEMENT OF LIVING AND WORKING CONDITIONS (Eurofound) (Hrsg.) (2018). Labour market change - State initiatives supporting the labour market integration of older workers - Not finished at 50: Keeping older workers in work.

European Lifelong Guidance Policy Network (ELGPN) (2015). Guidelines for Policies and Systems Development for Lifelong Guidance - A Reference for the EU and for the Commission. Tools No. 6.

European Lifelong Guidance Policy Network (ELGPN) (2015). Leitlinien für die Entwicklung politischer Strategien und Systeme lebensbegleitender Beratung -Ein Referenzrahmen für die Länder der Europäischen Union und für die Europäische Kommission. Tools No. 6.

Frey, A., ERtelt, B.-J. \& RupPeRT, J.-J. (2017). Berufsbiografien im Vergleich - Modul 7 für den Masterstudiengang Arbeitsmarktbezogene Beratung (M.A.). Mannheim: Hochschule der Bundesagentur für Arbeit.

Frey, A., Ertelt, B.-J., Ruppert, J.-J. (2018). Diagnosis of Social, Methodical, and Personal Competencies in Vocational Training and Job Analysis. A German Perspective. In: A. BIELA (ed.), European Questionnaire for Job Analysis (EQJA) - Theoretical and Methodological Bases (s. 101-127). Berlin: Peter Lang GmbH.

Froidevaux, A., Baumann, I., Maggiori, Chr., Weber, F., Rossier, J. (2017). Retirement Planning. How to Deal with Different Adjustment Trajectories? In: B.J. ERTELT, M. ScharpF (Hrsg.), Berufliche Beratung Älterer (s. 25-53). Frankfurt am Main: Peter Lang.

Graf, A. (2001). Lebensorientierte Personalentwicklung - Ein Ansatz für die Erhaltung und Förderung von Leistungsfähigkeit und - bereitschaft während des gesamten betrieblichen Lebenszyklus. Management, nr. 3, s. 24-31.

Graf, A. (2002). Lebenszyklusorientierte Personalentwicklung. Ein Ansatz für die Erhaltung und Förderung von Leistungsfähigkeit und -bereitschaft während des gesamten betrieblichen Lebenszyklus. Bern-Stuttgart-Wien.

Hafkesbrink, J., Krause, M. (2017). Age-Management - Den demografischen Wandel in Unternehmen gestalten. Freiburg-München-Stuttgart: Haufe Gruppe.

Herr, E.L., Cramer, St.H., Niles, Sp.G. (2004). Career Guidance and Counseling Through The Lifespan - Systematic Approaches. 6th ed. Boston: Pearson. 
Homrighausen, P., Wolf, K. (2018). Wiederbeschäftigungschancen Älterer - Wo Vermittlungsfachkräfte Handlungsbedarf sehen. Institut für Arbeitsmarkt- und Berufsforschung, IABKurzbericht, nr 11.

Lambeck, M. (2008). Lebensorientierte Personalarbeit - Die personalpolitische Antwort der DekaBank auf den demografischen Wandel. 54. IW-Studiengespräch zur beruflichen Bildung am 02.06.2008.

Maggiore, C., Nihil, R., Froidevaux, A., Rossier, J. (2014). Development and validation of the transition to retirement questionnaire. Journal of Career Assessment, 22, 505-523,

Mandl, I., Patrini, V., Jalava, J. Lantto, E. Muraille, M. (2018). Labour market change State initiatives supporting the labour market integration of older workers - Not finished at 50: Keeping older workers in work. European Foundation for the Improvement of Living and Working Conditions (Eurofound) (Hrsg.).

Nota, L., Rossier, J. (eds.) (2015). Handbook of Life Design - From Practice to Theory and From Theory to Practice. Göttingen: Hogrefe Publishing GmbH.

Organisation for ECONOMic Co-Operation and Development (OECD), European Communities (2004). Career Guidance - a Handbook for Policy Makers.

SAVICKAS, M.L. (2005). The theory and practice of career construction. In: S.D. BROWN, R.W. LENT (Eds.), Career development and counseling: Putting theory and research to work (s. 42-70). Hoboken, NJ: Wiley.

SAVickas, M.L. et al. (2011). Life-designing: Ein Paradigma für die berufliche Laufbahngestaltung im 21. Jahrhundert. Zeitschrift dvb-forum 1/2011 (Ertelt, B.-J., Ruppert, J.-J.: Deutsche Übersetzung und Anpassung des englischen Originalartikels 33-47).

Savickas, M.L., Nota, L., Rossier, J., Dauwalder, J.-P., Eduarda Duarte, M., Guichard, J., Soresi, S., VAN EsbroecK, R. \& VAN VianEN, A.E.M. (2009): Life designing: A paradigm for career construction in the 21 st century, Journal of Vocational Behavior, 75(3), 239-250.

SCHLOSSBerG, N.K. (2003). Retire smart, retire happy: Finding your true path in life. Washington DC: American Psychological Association.

Walker, J.W., Kimmel, D.C., \& Price, K.F. (1981): Retirement style and retirement satisfaction: Retirees aren't all alike. International Journal of Aging and Human Development, 12, $267-$ 281. In: Herr, Cramer, Niles 2004, s. 535.

\title{
BERUFLICHE BERATUNG FÜR MENSCHEN 50+ - UNTER BESONDERER BERÜCKSICHTIGUNG DES AGE-MANAGEMENTS IM HUMAN RESOURCE (HR)
}

\begin{abstract}
Berufsberatung für ältere Arbeitnehmer ist heute nicht nur ein wesentlicher Bestandteil des lebenslangen Lernens und Instrument der aktiven Arbeitsmarktpolitik in den EU-Ländern, sondern auch des AGE-Managements von Betrieben. Wichtig dabei ist die Orientierung an wissenschaftlichen Modellen der Lebensphasen, die sich nicht nur auf den beruflichen oder betrieblichen Bereich beziehen. Daher sind holistische Beratungsansätze, die auch die Lebensqualität der Berufstätigen berücksichtigen, von großer Bedeutung. Zur methodischen Gestaltung eigenen sich aus unserer Sicht die Lebensphasen- und Rollenvorstellungen sensu D. Super und ihre Weiterentwicklung durch Ansätze des „Life-Designing“ sensu M. Savickas et al. In dem Beitrag werden auch konkrete Vorschläge für ein altersgerechtes Human Resource Management gemacht.
\end{abstract}

Schlüsselwörter: Berufsberatung; AGE-Management; Lebensphasenmodelle; Übergangsberatung/Pre-Retirement; Beratungsbedarf. 


\title{
PROFESJONALNE DORADZTWO DLA OSÓB W WIEKU 50+ ZE SZCZEGÓLNYM UWZGLĘDNIENIEM ZARZĄDZANIA WIEKIEM W ZAKRESIE ZASOBÓW LUDZKICH (HR)
}

\begin{abstract}
Streszczenie
Doradztwo zawodowe dla starszych pracowników jest obecnie nie tylko niezbędnym elementem uczenia się przez całe życie i instrumentem aktywnej polityki rynku pracy w krajach UE, ale także zarządzania AGE w przedsiębiorstwach. Ważne jest, aby ukierunkować się na naukowe modele faz życia, które nie odnoszą się tylko do obszaru zawodowego lub firmy. Dlatego też bardzo ważne jest holistyczne podejście do poradnictwa, które uwzględnia również jakość życia osób pracujących. Z naszego punktu widzenia, fazy życia i koncepcje ról D. Super i ich dalszy rozwój poprzez podejście do projektowania życia (life design) M. Savickas et al. są odpowiednie dla metodycznego projektowania. W niniejszym artykule przedstawiono również konkretne propozycje dotyczące odpowiedniego do wieku zarządzania zasobami ludzkimi.
\end{abstract}

Słowa kluczowe: poradnictwo zawodowe; zarządzanie wiekiem (AGE); modele faz życia; poradnictwo przedemerytalne; potrzeby w zakresie poradnictwa.

\section{PROFESSIONAL COUNSELING FOR PEOPLE 50+ WITH SPECIAL CONSIDERATION OF THE AGE MANAGEMENT IN THE HUMAN RESOURCE (HR)}

\section{Summary}

Career guidance for older workers is today not only an essential part of lifelong learning and an instrument of active labour market policy in EU countries, but also of AGE management in enterprises. It is important to orientate oneself towards scientific models of the life phases, which do not only refer to the professional or company area. Therefore, holistic counselling approaches that also take into account the quality of life of working people are of great importance. In our view, the life phases and role concepts D. Super and their further development through approaches of "life design" M. Savickas et al. are suitable for methodical design. In this article, concrete proposals for age-appropriate human resource management are also made.

Key words: Career Guidance; AGE Management; Life Phase Models; Transitional Guidance/ Pre-Retirement; Guidance Needs. 